\title{
SUPERNOVA 1987A: LIGHT CURVES AND THEIR INTEPRETATION
}

\author{
R M Catchpole \\ South African Astronomical Observatory (SAAO) \\ P 0 Box 9, Observatory 7935 \\ Cape, South Africa
}

The Type II supernova SN1987A which occurred in the LMC is the brightest and most completely observed supernova ever recorded. Objective prism and UBV observations were made of the blue supergiant progenitor Sanduleak -690202 and indicate that the visual absorption lies in the range $0.4\left\langle A_{v}<0.6\right.$. Furthermore, the distance to the LMC is known in absolute units with a precision of about $\pm 15 \%(\mathrm{~m}-\mathrm{M}=$ 18.45, Feast 1988) which combined with the above data and subsequent photometric observations permits detailed comparison with theory.

Within 107 minutes of the Kamiokande IMB neutrino event the region of the supernova was being observed by Albert Jones, although it was not until 0.8 days after the event that the supernova was officially discovered by Shelton. The first photoelectric observation was made at 1.1 days, by William Allen (1988) a New Zealand amateur. Other observations made during the first two days, have been conveniently tabulated by Arnett (1988). During this time the supernova steadily brightened in $V$, although theory predicts that it was rapidly fading bolometrically and cooling, after the intense heating that occurred when the shock wave reached the stellar surface about 3 hours after core collapse.

As of August 1988, there is still a large body of unpublished photoelectric data so that it is premature to undertake a comprehensive review. This paper will be confined to a discussion of bolometric fluxes, based on the broad band photometry obtained by the CIIO/ESO and SAAO observers. The other major body of published UBVRI photometry is by the MSSSO observers (Dopita et al. 1988) and is in good agreement with the SAAO data. The relevant papers are listed in Table 1.

There is a large body of narrow band photometry in various systems which could prove valuable for comparison with theoretical model atmosphere calculations for the supernova. The narrow band widths of the filters make absolute flux calibration very much easier than for the broad band photometry.

The fine error sensor (FES) on the IUE satellite, freed as it is from clouds and seeing, gives excellent temporal coverage of SNI987A. However the FES sensitivity peaks near $B$ but stretches all the way to $9000 \AA$, which means that its effective wavelength will change in a complicated way as the $\mathrm{SN}$ evolves from a hot continuum to an emission line spectrum, making quantitative interpretation very difficult. 
Table 1

Photometry Papers discussed below

\section{CTIO}

Hamuy et al. 1988

Suntzeff et al. 1988

ESO

Bouchet et al. (1987a)

Cristiani et al. (1987)

Bouchet et al. (1988)

SAAO

Menzies et al. (1987)

Catchpole et al. (1987a)

Catchpole et al. (1987b)

Whitelock et al. (1988)

$$
\begin{aligned}
& \text { Days since } \\
& \text { core collapse }
\end{aligned}
$$

1 to 177
188 to 476

6 to 21

2 to 27

21 to 231



Photometric

Coverage
$U$ to $I$
$U$ to $I$

Fig. I shows the variation with time of the various broad band colours measured from SAAO. Note the rapid decline in the brightness at $U$ which is interpreted as partly due to the decreasing photospheris temperature and partly due to the dramatic increase in the UV line opacity. The effect in the IUE short wavelength bands was even more marked and their contribution to the bolometric flux can be safely ignored after the first few days. Also note the difference in linear decline rates at different wavelengths. Fig. 1 illustrates the

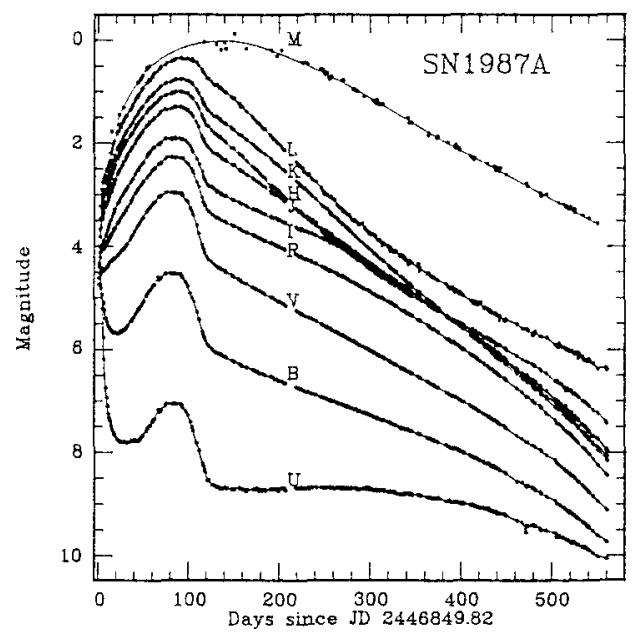

Fig. 1. The SAAO magnitudes as a function of time



Fig. 2. The bolometric magnitudes as a function of time 
importance of combining tic photomet, to form a bolometric curve rather than trying to compare individual magnitudes with models.

There are various ways in which the individual magnitudes, after conversion to fluxes, can be integrated to give the bolometric luminosity. In Fig. 2 the SAAO curve is based on a spline fit to $U$ to $M$ data where $M$ values have been taken from a smooth curve of $K-M$ against time. Also shown are magnitudes based on fluxes kindly supplied by Suntzeff and Bouchet (private communication) based on data obtained at ESO and CTIO. To allow comparison the SAAO bolometric magnitudes are calculated using $A_{v}=0.45$, the value adopted by ESO/CTI0 workers.

The agreement between the two data sets for the first 140 days is very close although during this time the ESO/CIIO results include SAAO J to $L$ photometry so that the two data sets are not entirely independent. Beyond day 140 differences arise between the two data sets largely because of differences in sensitivity in the I bands (Hamuy et al. 1988) at the two observatories. Menzies (1988) has shown that the wavelength sensitivity of the CTIO I band is much narrower than the SAAO band which results in the CTIO observers not including flux from the strong CalI emission lines at $8600 \AA$.

Two things are striking about the bolometric light curve, firstly it is quite unlike that of any other supernova in showing a second maximum and secondly it is very smooth on a time scale of a few days. Recently Young and Branch (1987) and Schmitz and Gaskell (1988) have pointed out examples of other supernovae that may have had similar light curves to SN1987A.

The difference between SN1987A and a more typical type, II if there is such a thing, is illustrated in Fig. 3 in a comparison with SN1969L, which after an initial rapid decline showed a distinct plateau phase before continuing to decline more slowly. The fact that the two SN light curves are very similar during the linear decline phase is probably fortuitous as we would expect Type II supernova progenitors to cover a wide range of masses with a possible corresponding range for the mass of ${ }^{56} \mathrm{Ni}$ created in the explosion.

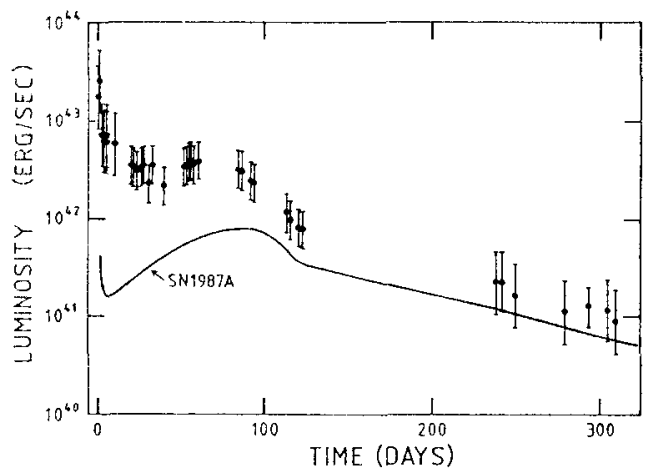

Fig. 3. SN19871 compared with SN1969L. The data is from Ciatti et al. (1971) \& Kirshner et al. (1973) as presented in Weaver \& Woosley (1980).

The unique and unexpected shape of the light curve, rapid colour evolution and the short time interval between the arrival of the neutrinos and visual brightening for 5N1987A are all apparently consequences of it arising from a compact blue, rather than an extended red giant, progenitor. 
The behaviour of the light curve can be divided into 5 phases which are illustrated symbolically in Fig. 4. Phase 4, which lasts for 143 days between days 122 and 265 after core collapse, shows a very closely linear decline in bolometric magnitude that in turn corresponds to an exponential decay in flux. The e-folding time of the decline depends on the method of integration and slightly on the adopted reddening but is very close to the 111.26 day mean life for radioactive decay of ${ }^{56}$ Co. Fig. 5 summarizes the e-folding times derived by different methods of integration and for different values of the reddening. The

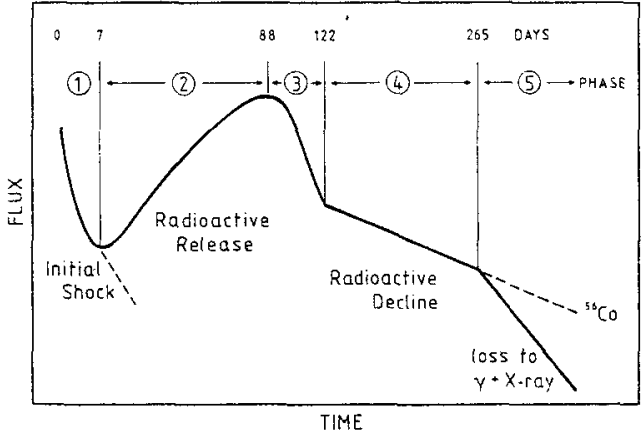

Fig. 4. Symbolic light-curve for SN1987A illustrating the 5 phases and their starting time in days. behaviour of individual colours is also shown. The slope of the bolometric curve can be thought of as the flux weighted mean of these individual colour slopes. The close agreement between the observed decline rates and the ${ }^{56}$ Co mean life provides convincing evidence that ${ }^{56} \mathrm{Co}$ and its progenitor: ${ }^{56} \mathrm{Ni}$ are the major sources of energy in the late time light curves of type II supernovae. This was predicted by Pankey (1962) and modelled for SN1987A by Woosley et al. (1988), Nomoto et al. (1988) and others. The Iight curve also indicates that any contribution from other energy sources such as a pulsar was not significant during phase 4. The phase 4 light curve can be used to calculate the mass of ${ }^{56 \mathrm{Ni}}$ produced in the initial explosion if we assume that only a negligible amount of the ${ }^{56}$ Co decay energy came out as $\gamma$ and $x$-rays. This assumption is justified by Kumagai et al. (1988) who show that $3 \%$ of the flux is emitted as $\gamma$ and $x$-rays at this time. For $A_{V}=0.6$ and a distance modulus $=18.5$ a mass of ${ }^{56} \mathrm{Ni}=0.08 \mathrm{MO}$ is derived. If we integrate the bolometric flux curve until day 265 and compare the total flux $\left(9.1 \times 10^{48} \mathrm{erg}\right)$ with that generated by radio-active decay in the same time interval we find the decay energy exceeds the radiated energy by $51 \%$ which is increased to $66 \%$ if we follow Woosley's theoretical prediction that up to day 40 the energy is derived from the initial shock wave. This excess energy is comparable to the kinetic energy of the ${ }^{56} \mathrm{Co}$ material and is probably responsible for mixing this material higher into the ejecta. This in turn can explain both the smooth shape of the light curve and the early appearance of $\gamma$ and $x-r$ ays (Nomoto et al. 1988). Once we have accepted that ${ }^{56} \mathrm{Ni}$ and ${ }^{56} \mathrm{Co}$ are major sources of energy we can then readily explain the remaining phases of the light curve. Phase 1 is defined by a rapid decline in brightness and redward change in all colour indices while during phase 2 the supernova slowly climbs to maximum brightness, something that at the time was totally unexplained and unexpected.

During phase 1 and well into phase 2 the flux distribution is well approximated by a blackbody which allows us to define a photospheric 




Fig.5. The e-folding time in days for various methods of integration and sets compared with ${ }^{56} \mathrm{Co}$ decay. The upper panel is for bolometric magnitudes, the lower panel for individual SAAO magnitudes.

radius and temperature. The variation of these with time are shown in Fig.6. Branch (1987) has shown that the density gradient within the photosphere can be deduced from the slope of the Log Radius against Log Time curve. The slope changes on day 5 and corresponds to a change of density from $\rho \simeq R^{-11.7}$ to $\rho \sim R^{-5.0}$. The definition of a photosphere combined with radial velocity measurements of faint lines formed at the photosphere allow the distance of SN1987A to be calculated. Using

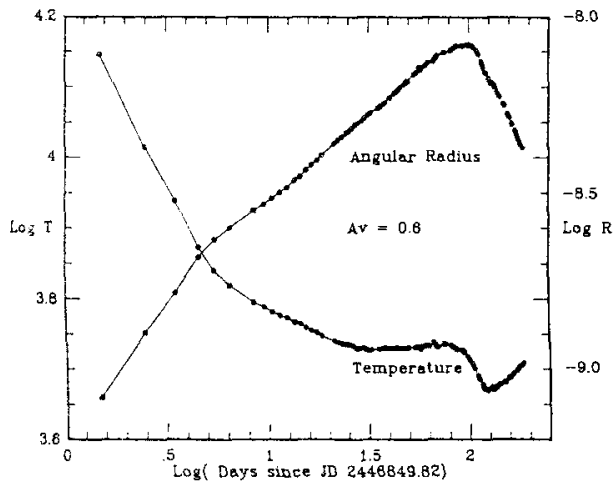

Fig.6. Variation of blackbody temperature and radius with time from the SAAO data. this method Branch (1987) derives a modulus of $18.7 \pm 0.2$ in good agreement with the generally adopted value of $18.5 \pm 0.15$, demonstrating the validity of the method for distance determination. The temperature initially declines rapidly and then remains constant at the value appropriate to that of $H$ recombination showing that the position of the photosphere is defined by the sharp increase in opacity as one crosses from the neutral to ionized hydrogen.

The decline in brightness during phase 1 , that lasts until day 7 , is caused by the rapid decline in photospheric temperature being more important than the increase in curve enters phase 2 and the $5 N$ 
starts to brighten the increasing radius of the photosphere more than compensates for the much slower decrease in temperature. Woosley et al. (1988) predict that for about the first 40 days the $5 \mathrm{~N}$ is entirely powered by energy deposited in the outer envelope by the passage of the initial shock wave. Thereafter the steady rise in brightness to maximum light on day 88 is a consequence of the photosphere which, although expanding in length coordinates, is falling back in mass coordinates and progressively liberating radiation generated by radioactive decay. The slope of the light curve during phase 2 and the time of maximum brightness both impose constraints on models of the explosion in terms of the degree of mixing, the mass of the expanding envelope and the energy of the explosion.

During Phase 3 , from day 88 to day 122 , the brightness declined steeply. It was during this time that an excess of flux first appeared at $4.8 \mu \mathrm{m}$ and rapidly increased in strength. Longer wavelength photometry indicated that this excess was confined to the $M$ band and it was identified with emission from the fundamental vibration rotation band of $\mathrm{CO}$. The width and velocity of the $\mathrm{CO}$ lines showed that they arose in the envelope and indicated that the excess flux should be added into the current energy budget of the SN. In some supernovae an infrared excess has been observed and attributed to a light echo of the original UV flash. In which case the energy should not be included in the current energy budget. In SNI987A a weak excess of flux beyond $4 \mu \mathrm{m}$, of uncertain origin, was noted by Bouchet et al. (1987b) as early as day 59 but amounted to less than $1 \%$ of the total flux.

Phase 5 which commenced on day 265, corresponds to an increasingly rapid decline in brightness in the $U$ to $M$ bolometric flux as more of the ${ }^{56}$ Co energy escapes as $\gamma$ rays and $x$-rays.

The CIIO/ESO data is not in complete agreement with this interpretation as their curve always falls below the SAAO curve during the linear decline and does not show a change of slope between phases 4 and 5. The differences are more clearly illustrated in Fiq. 7 where the SAAO and CTIO/ESO data are compared directly with the ${ }^{56} \mathrm{Co}$ e-folding

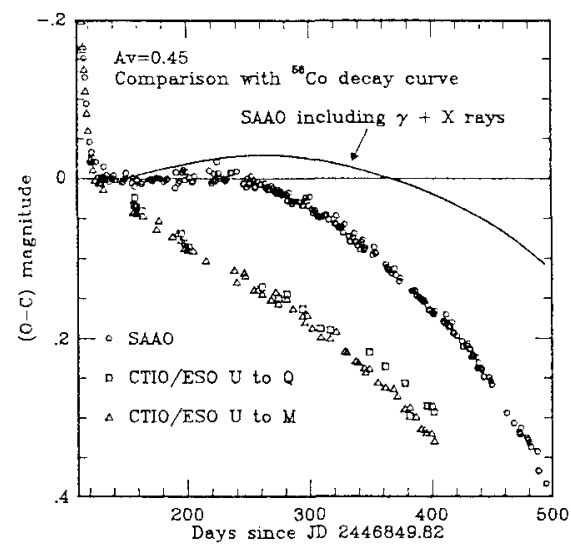

Fig. 7. Differences between the CTIO/ESO and SAAO bolometric magnitudes and the ${ }^{56}$ Co decay curve are given as a function of time. The continuous curve shows the effect of adding the $\gamma$ and $X$-ray flux to the SAAO curve.

curve ( 111.26 day). The CIIO/ESO observers show two curves one for $U$ to $M(0.3$ to $4.8 \mu \mathrm{m})$ and one for $U$ to $Q(0.3$ to $20 \mu \mathrm{m})$. The similarity 
of the two slopes shows the discrepancy does not arise from the failure of SAAD to include data out to $20 \mu \mathrm{m}$. About 0.05 of the difference between the SAAO and ESO/CTIO residuals may be caused by the different methods of inteqration used at the two observatories while the main difference arises from differences in the $I$ band discussed above.

In order to determine the true bolometric flux from the supernova all the flux from outside the $U$ to $M$ region must be included. Up to the present (August 1988) the only other important source of energy is in the $\gamma$ and $x$-rays. X-rays in the $16-30 \mathrm{keV}$ range have been detected from 5N1987A since day 130 and ${ }^{56} \mathrm{Co}$ line emission at $547 \mathrm{keV}$ and $1238 \mathrm{keV}$ has been measured since about day 180. Unfortunately before this data can be combined with the $U$ to $M$ data we must include the Compton scattered but usually unobserved flux between 16 and $847 \mathrm{keV}$ which must be modelled. This has been done by Kumagai et al. (1988) and the results of adding their predicted fluxes to the SAAO data are also shown in Fig. 7. This has the effect of reducing the deviation of the CTIO data from the ${ }^{56}$ Co decay curve while the SAAO data now lies within \pm 3\% of the curve for 300 days after day 140. In view of quite large uncertainties in the $\gamma$ and $x$-ray data the overall agreement is rather satisfactory.

Uncertainties in determining the absolute bolometric flux can be divided into astrophysical and observational uncertainties. The main astrophysical uncertainties are in the distance modulus and interstellar reddening. The problem of whether or not to include any infrared excess in the energy balance has not yet arisen. The two major observational problems are undoubtedly the incompleteness of coverage of the spectrum by the broad bands and the choice of the most appropriate integration technique. In the early days, when the supernova still showed a strong continuum, interpolation between adjacent bands introduced relatively small errors. As the spectrum shows more emission lines and the continuum fades the problem becomes much worse and it is possible for individual lines to lie between bands and not be measured at all. The omission of Paschen $\alpha$ at $1.876 \mu \mathrm{m}$ between the $H$ and $K$ bands may have caused the bolometric flux to be underestimated by a few percent from day 200. Also the band heads of the co fundamental band lie outside to blue of the $M$ band. As more spectrophotometry becomes available it should be possible to improve our knowledge of the bolometric curve. However it must be emphasized that this will be an iterative procedure as spectrophotometry also has calibration problems. other observational problems are an accurate knowledge of both the filter transmissions and their absolute flux calibrations.

Future photometric possibilities include possible dust formation, evidence for a pulsar and the search for further evidence of light echoes.

I am indebted to the entire $S A A O$ staff and visitors who have contributed to observations of SN1987A and to Stan Woosley, Ken Nomoto and David Arnett for their enlightening preprints on which much of the theoretical discussion here is based. 


\section{REFERENCES}

Allen, $W H$, 1988. Publ. Var. Star Sect., RASNZ.

Arnett, W D, 1988. Astrophys. J., 331, 377.

Bouchet, P, Moneti, A, Slezak, E, Le Bertre, T, \& Manfroid, J, 1988. ESO preprint No. 592.

Bouchet, P, Stanga, R \& Le Bertre, T, 1987a. Astr. Astrophys., 177,L9.

Bouchet, P, Stanga, R, Moneti, A, Le Bertre, T, Manfroid, J, Silvestro, $G$ \& Slezak, E, 1987b. ESO Workshop on the SN1987A. ESO Conference and Workshop Proceedings No. 26, p.79, ed. Danziger, I J, Garching.

Branch, D, 1987. Astrophys. J., 320, L23.

Catchpole, R M \& Menzies, J W et al., 1987a. Mon. Not. R. astr. Soc., $229,15 p$.

Catchpole, R M, Whitelock, P A \& Feast, M W et al., 1987b. Mon. Not. R. astr. Soc., 231, 75p.

Ciatti, F, Rosino, L, \& Bertola, F, 1971. Mem. Soc. Astr. Ital. 42, 163.

Cristiani, S, Babel, J \& Barwig, H, 1987. Astr. Astrophys., 177, L5. Dopita, M A, \& Dawe, J A, et al. 1988. Astr. J., 95, 1717.

Feast, $M$ W, 1988. SAAO preprint No. 599.

Hamuy, M, Suntzeff, N B, Gonzalez, R, \& Martin, G, 1988. Astr. J., $95,63$.

Kirshner, R P, Oke, J B, Penston, M V, \& Searle, L, 1973. Astrophys. J. , 185,303 .

Kumagāi S, Itoh, M, Shigeyama, T, Nomoto, K, \& Nishimura, J, 1988. Astr. Astrophys., 197, L7.

Menzies, J W, 1988. SAAO preprint No. 598.

Menzies, J W \& Catchpole, R M et al. Mon. Not. R. astr. Soc., 227, 39p. Nomoto, K, Shigeyama, $T$ \& Hashimoto, $M$, 1988. Lecture Notes in Physics, 305, 319.

Pankey Jr, T, $\overline{1962}$. Ph.D. Thesis, Howard University, Washington DC.

Schmitz, M F \& Gaskell, C M, 1988. Proc. of the fourth George Mason Astrophysics Workshop, p.112, ed. Kafatos, M \& Michalitsianos, A G. Cambridge University Press.

Suntzeff, N B, Hamuy, M, Martin, G, Garnez, A, \& Gonzalez, R, 1988 preprint.

Weaver, T A, \& Woosley, S E, 1980. Ann. NY Acad. Sci., 336, 335.

Whitelock, $P$ A, Catchpole, $R$ M, Menzies, J W \& Feast, $M W$, et al., 1988. Mon. Not. R. astr. Soc., in press.

Woosley, S E, Pinto, P A \& Ensman, L, 1988. Astrophys. J. , 324, 466.

Young, T R \& Branch, D, 1987. Nature, 333, 305. 